PROCEEDINGS OF THE

AMERICAN MATHEMATICAL SOCIETY

Volume 134, Number 9, September 2006, Pages 2503-2510

S 0002-9939(06)08269-4

Article electronically published on February 17, 2006

\title{
DEFORMATIONS AND DERIVED EQUIVALENCES
}

\author{
FRAUKE M. BLEHER
}

(Communicated by Martin Lorenz)

\begin{abstract}
Suppose $A$ and $B$ are block algebras of finite groups over a complete local commutative Noetherian ring whose residue field is a field $k$ of positive characteristic. We prove that a split-endomorphism two-sided tilting complex (as introduced by Rickard) for the derived categories of bounded complexes of finitely generated modules over $A$, resp. $B$, preserves the versal deformation rings of bounded complexes of finitely generated modules over $k A$, resp. $k B$.
\end{abstract}

\section{INTRODUCTION}

Suppose $k$ is a field of characteristic $p>0, W$ is a complete local commutative Noetherian ring with residue field $k$, and $G$ is a profinite group. In [11, Mazur developed a deformation theory of finite-dimensional representations of $G$ over $k$, using work of Schlessinger [17. A more explicit approach was later described by de Smit and Lenstra in [8]. In the meantime, deformation theory has become a basic tool in arithmetic algebraic geometry (see e.g. [7, [19, 18, [5] and their references). In 3, 4, the author and Chinburg generalized Mazur's deformation theory to objects of bounded complexes of pseudocompact modules over the completed group algebra $[[k G]]$ (see Section 3 for definitions in case $G$ is finite).

One motivation for [3, 4, arose from the study of universal deformation rings for finite groups. In [2] and [1, Morita equivalences between module categories were a basic tool in determining universal deformation rings of representations associated to blocks with cyclic or Klein four defect groups. Since Morita equivalences cannot be expected to exist in a broad context, one is led to consider other types of equivalences. Conjectures of Broué and others (see e.g. [6, 15, and their references) would establish derived equivalences between various blocks of group algebras of finite groups. For example, Broué conjectured that if a finite group $G$ has an abelian Sylow $p$-subgroup $D$, then the principal blocks of $W G$ and of $W N_{G}(D)$ have equivalent derived categories (in the case $W$ is a complete discrete valuation ring of characteristic zero). In [12, Rickard proved that two block algebras over $W$ are derived equivalent if and only if there are so-called two-sided tilting complexes for the blocks. He further showed that these two-sided tilting complexes can always be

Received by the editors May 7, 2004 and, in revised form, March 27, 2005.

2000 Mathematics Subject Classification. Primary 20C05; Secondary 18 E30.

Key words and phrases. Universal deformations, versal deformations, derived categories, derived equivalences, abelian defect group conjecture, tilting complexes.

The author was supported in part by NSA Young Investigator Grant MDA904-01-1-0050 and NSF Grant DMS01-39737.

(C)2006 American Mathematical Society Reverts to public domain 28 years from publication 
chosen to have a split-endomorphism property (see e.g. 13, 14]). If one wants to ensure that these tilting complexes are also compatible with the $p$-local structures of the block algebras, one needs to require additional conditions to obtain so-called splendid tilting complexes (see [13] for principal blocks and 10] for general blocks).

The idea is to use such derived equivalences to simplify the determination of (uni)versal deformation rings of representations or bounded complexes by considering smaller groups. Thus one needs that derived equivalences preserve (uni)versal deformation rings of bounded complexes. In this paper, we consider derived equivalences given by split-endomorphism two-sided tilting complexes. Our main result is Theorem 3.2 which proves that a split-endomorphism two-sided tilting complex for two blocks $A$ and $B$ of finite groups over $W$ induces an isomorphism between the versal deformation ring of a bounded complex of finitely generated $k A$-modules and the corresponding complex of $k B$-modules. A key ingredient in the proof is that two-sided tilting complexes behave well when extending scalars from $W$ to a complete local commutative Artinian $W$-algebra $R$ with residue field $k$ (see [12] and Lemma 2.1).

The paper is organized as follows. In Section 2, we recall the necessary background about split-endomorphism two-sided tilting complexes. In Section 3, we give the basic definitions and results about deformations of objects of bounded complexes of pseudocompact modules over $k G$ in case $G$ is a finite group. We also prove our main result Theorem [3.2. In Section 4 we consider the example of split-endomorphism two-sided tilting complexes for the principal blocks of the alternating groups $A_{4}$ and $A_{5}$ in characteristic 2.

\section{TWO-SIDED TILTING COMPLEXES AND SCALAR EXTENSIONS}

Suppose $k$ is a field of positive characteristic $p$ and $W$ is a complete local commutative Noetherian ring with residue field $k$. Define $\hat{\mathcal{C}}$ to be the category of complete local commutative Noetherian $W$-algebras with residue field $k$. The morphisms in $\hat{\mathcal{C}}$ are continuous $W$-algebra homomorphisms which induce the identity on $k$. Let $\mathcal{C}$ be the full subcategory of Artinian objects in $\hat{\mathcal{C}}$.

Suppose $G$ (resp. $H$ ) is a finite group, and let $A$ (resp. $B$ ) be a block of $W G$ (resp. $W H$ ). Then $A$ and $B$ are both $W$-algebras that are projective as $W$ modules. Moreover, $A$ and $B$ are symmetric $W$-algebras in the sense that $A$ (resp. $B)$ is isomorphic to its $W$-linear dual $\check{A}=\operatorname{Hom}_{W}(A, W)\left(\right.$ resp. $\left.\check{B}=\operatorname{Hom}_{W}(B, W)\right)$ as an $A$-A-bimodule (resp. $B$ - $B$-bimodule). For $R \in \mathrm{Ob}(\hat{\mathcal{C}})$, define $R A$ (resp. $R B$ ) to be the block algebra in $R G$ (resp. $R H$ ) corresponding to $A$ (resp. $B$ ), i.e. $R A=R \otimes_{W} A$ (resp. $R B=R \otimes_{W} B$ ).

If $S$ is a ring, $S$-mod denotes the category of finitely generated left $S$-modules. Let $C^{b}\left(S\right.$-mod) be the category of bounded complexes in $S$-mod, let $K^{b}(S$-mod) be the homotopy category of $C^{b}(S$-mod $)$, and let $D^{b}(S$-mod) be the derived category of $K^{b}(S$-mod).

Rickard proved in [12] (see also [13, §2] and [14, §9.2]) that the derived categories $D^{b}(A$-mod $)$ and $D^{b}(B$-mod $)$ are equivalent as triangulated categories if and only if there is a bounded complex $P^{\bullet}$ of finitely generated $A$-B-bimodules and a bounded complex $Q^{\bullet}$ of finitely generated $B$ - $A$-bimodules such that

$$
\begin{array}{ll}
P^{\bullet} \otimes_{B}^{\mathbf{L}} Q^{\bullet} \cong A & \text { in } D^{b}\left(\left(A \otimes_{W} A^{o p}\right) \text {-mod }\right), \text { and } \\
Q^{\bullet} \otimes_{A}^{\mathbf{L}} P^{\bullet} \cong B & \text { in } D^{b}\left(\left(B \otimes_{W} B^{o p}\right) \text {-mod }\right) .
\end{array}
$$


If $P^{\bullet}$ and $Q^{\bullet}$ exist, then the functors

$$
\begin{aligned}
& P^{\bullet} \otimes_{B}^{\mathbf{L}}-: D^{b}(B \text {-mod }) \rightarrow D^{b}(A \text {-mod }) \quad \text { and } \\
& Q^{\bullet} \otimes_{A}^{\mathbf{L}}-: D^{b}(A \text {-mod }) \rightarrow D^{b}(B \text {-mod })
\end{aligned}
$$

are equivalences of derived categories, and $Q^{\bullet}$ is isomorphic to $\mathbf{R H o m}_{A}\left(P^{\bullet}, A\right)$ in the derived category of $B$-A-bimodules. The complexes $P^{\bullet}$ and $Q^{\bullet}$ are called twosided tilting complexes. Moreover, Rickard showed (see [13, §2] and [14, §9.2.2]) that we may assume that all terms of $P^{\bullet}$ are projective as left $A$-modules and as right $B$-modules and that all but one of the terms are actually projective as $A$ $B$-bimodules. Since $A$ is a symmetric $W$-algebra, the functors $\operatorname{Hom}_{A}(-, A)$ and $\operatorname{Hom}_{W}(-, W)$ are naturally isomorphic, and so we may take $Q^{\bullet}$ to be the linear dual $\check{P}^{\bullet}=\operatorname{Hom}_{W}\left(P^{\bullet}, W\right)$ of $P^{\bullet}$. In this situation, (2.1) is equivalent to

$$
\begin{array}{ll}
A \cong P^{\bullet} \otimes_{B} \check{P}^{\bullet} \cong \operatorname{Hom}_{B}\left(P^{\bullet}, P^{\bullet}\right) & \text { in } K^{b}\left(\left(A \otimes_{W} A^{o p}\right) \text {-mod }\right), \text { and } \\
B \cong \check{P}^{\bullet} \otimes_{A} P^{\bullet} \cong \operatorname{Hom}_{A}\left(P^{\bullet}, P^{\bullet}\right) & \text { in } K^{b}\left(\left(B \otimes_{W} B^{o p}\right) \text {-mod }\right) .
\end{array}
$$

Rickard calls a bounded complex $P^{\bullet}$ of finitely generated $A$-B-bimodules a splitendomorphism two-sided tilting complex, if all terms of $P^{\bullet}$ are projective as left and as right modules and (2.3) is satisfied.

The following lemma follows from [12, Thm. 2.1].

Lemma 2.1. Suppose $A, B$ are block algebras as above, $R \in \mathrm{Ob}(\mathcal{C})$ is Artinian, and $P^{\bullet}$ is a split-endomorphism two-sided tilting complex in $D^{b}\left(\left(A \otimes_{W} B^{o p}\right)\right.$-mod). Then

$$
P_{R}^{\bullet}=R \otimes_{W} P^{\bullet}
$$

is a split-endomorphism two-sided tilting complex in $D^{b}\left(\left(R A \otimes_{R} R B^{o p}\right)\right.$-mod $)$.

\section{TWO-SIDED TILTING COMPLEXES AND DEFORMATIONS}

Keeping the notation of the previous section, we want to use Lemma 2.1 to prove that split-endomorphism two-sided tilting complexes preserve versal deformation rings. We first remind the reader of the basic definitions of quasi-lifts and deformations of complexes that were introduced in [3, 4,

Let $R \in \mathrm{Ob}(\hat{\mathcal{C}})$. Note that since $G$ is assumed to be a finite group, the abstract group algebra $R G$ is the same as the completed group algebra [[RG]]. An $R G$ module $M$ is said to be pseudocompact, if it is the projective limit of $R G$-modules of finite length having the discrete topology. In particular, every finitely generated $R G$-module is pseudocompact. Let $C^{-}(\operatorname{PCMod}(R G))$ be the abelian category of bounded above complexes of pseudocompact $R G$-modules, let $K^{-}(\operatorname{PCMod}(R G))$ be the corresponding homotopy category, and let $D^{-}(\operatorname{PCMod}(R G))$ be the corresponding derived category. We say that a complex $M^{\bullet}$ in $K^{-}(\operatorname{PCMod}(R G))$ has finite pseudocompact $R$-tor dimension, if there exists an integer $N$ such that for all pseudocompact $R$-modules $S$, and for all integers $i<N, \mathrm{H}^{i}\left(S \hat{\otimes}_{R}^{\mathbf{L}} M^{\bullet}\right)=0$. Here $\hat{\otimes}_{R}$ denotes the completed tensor product in the category of pseudocompact $R$-modules. Note that if $M$ is finitely generated as pseudocompact $R$-module, then the functors $M \otimes_{R}-$ and $M \hat{\otimes}_{R}-$ are naturally isomorphic.

Definition 3.1. Let $V^{\bullet}$ be a complex in $D^{-}(\operatorname{PCMod}(k G))$ which has only finitely many non-zero cohomology groups, all of which have finite $k$-dimension. A quasilift of $V^{\bullet}$ over an object $R$ of $\hat{\mathcal{C}}$ is a pair $\left(M^{\bullet}, \phi\right)$ consisting of a complex $M^{\bullet}$ in $D^{-}(\operatorname{PCMod}(R G))$ which has finite pseudocompact $R$-tor dimension together with 
an isomorphism $\phi: k \hat{\otimes}_{R}^{\mathbf{L}} M^{\bullet} \rightarrow V^{\bullet}$ in $D^{-}(\operatorname{PCMod}(k G))$. Two quasi-lifts $\left(M^{\bullet}, \phi\right)$ and $\left(M^{\prime \bullet}, \phi^{\prime}\right)$ are isomorphic if there exists an isomorphism $M^{\bullet} \rightarrow M^{\prime \bullet}$ in $D^{-}(\operatorname{PCMod}(R G))$ which carries $\phi$ to $\phi^{\prime}$. A deformation of $V^{\bullet}$ over $R$ is an isomorphism class of quasi-lifts of $V^{\bullet}$.

The deformation functor $\hat{F}_{V} \bullet: \hat{\mathcal{C}} \rightarrow$ Sets associated to $V^{\bullet}$ is defined as follows. It sends an object $R$ of $\hat{\mathcal{C}}$ to the set $\hat{F}_{V} \bullet(R)$ of all deformations of $V^{\bullet}$ over $R$, and it sends a morphism $\alpha: R \rightarrow R^{\prime}$ in $\hat{\mathcal{C}}$ to the set map $\hat{F}_{V} \bullet(R) \rightarrow \hat{F}_{V} \bullet\left(R^{\prime}\right)$ induced by $M^{\bullet} \mapsto R^{\prime} \hat{\otimes}_{R, \alpha}^{\mathrm{L}} M^{\bullet}$. Let $F_{V} \bullet$ be the restriction of $\hat{F}_{V} \bullet$ to the subcategory $\mathcal{C}$ of Artinian objects in $\hat{\mathcal{C}}$.

It was proved in [4, Thm. 2.14] that $F_{V} \bullet$ has a pro-representable hull $R\left(G, V^{\bullet}\right) \in$ $\operatorname{Ob}(\hat{C})$ in the sense of [17, Def. 2.7], and that $\hat{F}_{V} \bullet$ is continuous. The $\operatorname{ring} R\left(G, V^{\bullet}\right)$ is called the versal deformation ring of $V^{\bullet}$. Moreover, if $\operatorname{Hom}_{D^{-}(k G)}\left(V^{\bullet}, V^{\bullet}\right)=k$, then $\hat{F}_{V} \bullet$ is represented by $R\left(G, V^{\bullet}\right)$, in which case $R\left(G, V^{\bullet}\right)$ is called the universal deformation ring of $V^{\bullet}$.

Theorem 3.2. Suppose $A, B$ are block algebras as above, and $Q^{\bullet}$ is a split-endomorphism two-sided tilting complex in $D^{b}\left(\left(B \otimes_{W} A^{o p}\right)\right.$-mod $)$. Let $V^{\bullet}$ be a bounded complex of finitely generated $k A$-modules, and let $V^{\mathbf{\prime}^{\bullet}}=\left(k \otimes_{W} Q^{\bullet}\right) \otimes_{k A} V^{\bullet}$. Then $R\left(G, V^{\bullet}\right)$ and $R\left(H, V^{\mathbf{\prime}^{\bullet}}\right)$ are isomorphic.

Proof. Suppose $R \in \mathrm{Ob}(\mathcal{C})$ is Artinian, and let $\left(M^{\bullet}, \phi\right)$ be a quasi-lift of $V^{\bullet}$ over $R$.

Claim 1. The quasi-lift $\left(M^{\bullet}, \phi\right)$ is isomorphic to a quasi-lift $\left(N^{\bullet}, \psi\right)$ of $V^{\bullet}$ over $R$, where $N^{\bullet}$ and $\psi$ are in $D^{b}\left(R A\right.$-mod), such that the terms of $N^{\bullet}$ are abstractly free as $R$-modules.

Proof of Claim 1. By [4, Cor. 3.6], we may assume without loss of generality that $M^{\bullet}$ is a bounded-above complex of finitely generated abstractly free $R G$-modules. Then $k \hat{\otimes}_{R}^{\mathbf{L}} M^{\bullet}=k \otimes_{R} M^{\bullet}$ is also a bounded above complex of finitely generated abstractly free $k G$-modules, and we may assume that $\phi$ is given by a morphism in $C^{-}(\operatorname{PCMod}(k G))$. Let $e$ be the idempotent corresponding to the block $A$. Then $M^{\bullet}=e M^{\bullet} \oplus(1-e) M^{\bullet}$ and $\phi=e \phi \oplus(1-e) \phi$. Since $M^{\bullet}$ has finite pseudocompact $R$-tor dimension and since the functor $\mathrm{H}^{n}$ is additive for all $n, e M^{\bullet}$ and $(1-e) M^{\bullet}$ also have finite pseudocompact $R$-tor dimension. Hence $\left(e M^{\bullet}, e \phi\right)$ is a quasi-lift of $e V^{\bullet}=V^{\bullet}$, and $\left((1-e) M^{\bullet},(1-e) \phi\right)$ is a quasi-lift of the zero complex $(1-e) V^{\bullet}$. It follows that the quasi-lift $\left(M^{\bullet}, \phi\right)$ is isomorphic to the quasi-lift $\left(e M^{\bullet}, e \phi\right)$ of $V^{\bullet}$. Since $e M^{\bullet}$ has finite pseudocompact $R$-tor dimension and since the terms of $e M^{\bullet}$ are finitely generated abstractly free $R$-modules, we can truncate $e M^{\bullet}$ to obtain a quasi-lift $\left(N^{\bullet}, \psi\right)$ of $V^{\bullet}$ which is isomorphic to $\left(e M^{\bullet}, e \phi\right)$ such that $N^{\bullet}$ is a bounded complex of finitely generated $R A$-modules, all of which are abstractly free as $R$-modules. This completes the proof of Claim 1.

Claim 2. Suppose $R^{\prime} \in \operatorname{Ob}(\mathcal{C})$ is Artinian, and $\alpha: R \rightarrow R^{\prime}$ is a morphism in $\mathcal{C}$. Let $\pi: R \rightarrow k$, resp. $\pi^{\prime}: R^{\prime} \rightarrow k$, be the canonical surjection, and let $Q_{R}^{\bullet}=R \otimes_{W} Q^{\bullet}$ and $Q_{R^{\prime}}^{\bullet}=R^{\prime} \otimes_{W} Q^{\bullet}$. Then for each complex $X^{\bullet}$ in $D^{b}(R A$-mod), there is an isomorphism

$$
h_{R, R^{\prime}, \alpha}^{X}:\left(Q_{R}^{\bullet} \otimes_{R A} X^{\bullet}\right) \otimes_{R, \alpha} R^{\prime} \rightarrow Q_{R^{\prime}}^{\bullet} \otimes_{R^{\prime} A}\left(X^{\bullet} \otimes_{R, \alpha} R^{\prime}\right)
$$


in the category of bounded complexes of left $R^{\prime} B$-modules such that

$$
h_{R^{\prime}, k, \pi^{\prime}}^{X \bullet R_{R}^{\prime}} \circ\left(h_{R, R^{\prime}, \alpha}^{X \bullet} \otimes k\right)=h_{R, k, \pi}^{X \bullet}
$$

when we identify

$$
\left(\left(Q_{R}^{\bullet} \otimes_{R A} X^{\bullet}\right) \otimes_{R, \alpha} R^{\prime}\right) \otimes_{R^{\prime}} k=\left(Q_{R}^{\bullet} \otimes_{R A} X^{\bullet}\right) \otimes_{R} k
$$

and

$$
\left(X^{\bullet} \otimes_{R, \alpha} R^{\prime}\right) \otimes_{R^{\prime}} k=X^{\bullet} \otimes_{R} k .
$$

Proof of Claim 2. Suppose first that $Q$ is a finitely generated $B$ - $A$-bimodule which is projective as right $A$-module, and suppose $X$ is a finitely generated left $R A$ module. Then the map

$$
\begin{aligned}
h_{R, R^{\prime}, \alpha}^{Q, X}:\left(\left(R \otimes_{W} Q\right) \otimes_{R A} X\right) \otimes_{R, \alpha} R^{\prime} & \rightarrow\left(R^{\prime} \otimes_{W} Q\right) \otimes_{R^{\prime} A}\left(X \otimes_{R, \alpha} R^{\prime}\right) \\
((r \otimes q) \otimes x) \otimes r^{\prime} & \mapsto(\alpha(r) \otimes q) \otimes\left(x \otimes r^{\prime}\right)
\end{aligned}
$$

is an $R^{\prime} B$-module isomorphism which is natural in both variables $Q$ and $X$. It follows from the definition of the tensor product complex $Q_{R}^{\bullet} \otimes_{R A} X^{\bullet}$ that there is an isomorphism in $C^{b}\left(R^{\prime} B\right.$-mod) as stated in (3.1). Since $\alpha$ induces the identity on $k$, one easily checks (3.2), which proves Claim 2 .

It follows from Lemma 2.1 that $Q_{R}^{\bullet}=R \otimes_{W} Q^{\bullet}$ is a split-endomorphism two-sided tilting complex in $D^{b}\left(\left(R B \otimes_{W} R A^{o p}\right)\right.$-mod $)$. Let $\left(N^{\bullet}, \psi\right)$ be as in Claim 1 , and define $N^{\prime}=Q_{R}^{\bullet} \otimes_{R A} N^{\bullet}$. Since the terms of $Q_{R}^{\bullet}$ are finitely generated projective right $R A$-modules and the terms of $N^{\bullet}$ are finitely generated abstractly free $R$-modules, it follows that the terms of $N^{\prime^{\bullet}}=Q_{R}^{\bullet} \otimes_{R A} N^{\bullet}$ are finitely generated projective, and hence abstractly free, $R$-modules. Because $N^{\prime \bullet}$ is a bounded complex, this means that $N^{\prime \bullet}$ has finite pseudocompact $R$-tor dimension. Moreover,

$$
N^{\prime \bullet} \hat{\otimes}_{R}^{\mathbf{L}} k=N^{\prime \bullet} \otimes_{R} k=\left(Q_{R}^{\bullet} \otimes_{R A} N^{\bullet}\right) \otimes_{R} k \stackrel{h_{R, k, \pi}^{N^{\bullet}}}{\longrightarrow} Q_{k}^{\bullet} \otimes_{k A}\left(N^{\bullet} \otimes_{R} k\right) \stackrel{Q_{k}^{\bullet} \otimes \psi}{\longrightarrow} V^{\prime \bullet}
$$

is an isomorphism in $D^{b}\left(k B\right.$-mod). This implies that $\left(N^{\prime \bullet},\left(Q_{k}^{\bullet} \otimes \psi\right) \circ h_{R, k, \pi}^{N^{\bullet}}\right)$ is a quasi-lift of $V^{\prime \bullet}$ over $R$. We therefore obtain for all $R \in \mathrm{Ob}(\mathcal{C})$ a bijection $\tau_{R}$ from the set of deformations of $V^{\bullet}$ over $R$ onto the set of deformations of $V^{\prime \bullet}$ over $R$. Using (3.1) and (3.2) from Claim 2, it follows that the $\tau_{R}$ are natural with respect to homomorphisms $\alpha: R \rightarrow R^{\prime}$ in $\mathcal{C}$. Since the deformation functors $\hat{F}_{V} \bullet$ and $\hat{F}_{V^{\prime}}$ are continuous, this implies that they are naturally isomorphic. Hence the versal deformation rings $R\left(G, V^{\bullet}\right)$ and $R\left(H, V^{\prime \bullet}\right)$ are isomorphic. This completes the proof of Theorem 3.2

\section{The PRINCIPAL BLOCKS OF $A_{4}$ AND $A_{5}$ IN CHARACTERISTIC 2}

In this section, we want to look at the following example. Let $k$ be an algebraically closed field of characteristic 2, and let $W$ be the ring of infinite Witt vectors over $k$. Let $A_{4}$, resp. $A_{5}$, denote the alternating group on 4, resp. 5, letters. Then $W A_{4}$ is its own principal block, and we denote the principal block of $W A_{5}$ by $B$. Rickard showed in [13, §3] (see also [16, Example 1]) that there is a split-endomorphism two-sided tilting complex $Q_{k}^{\bullet}$ for $k B$ and $k A_{4}$. In fact, Rickard showed that this complex is a so-called splendid tilting complex, and can thus be lifted to a splendid tilting complex $Q^{\bullet}$ for $B$ and $W A_{4}$ [13, Thm. 5.2]. We now describe the tilting complex $Q_{k}^{\bullet}$, as given in [13, §3]. We identify $A_{4}$ with the normalizer of a Sylow 2-subgroup of $A_{5}$. 
The group algebra $k A_{4}$ has three simple modules: the trivial simple module $S_{0}$, and two other one-dimensional modules $S_{1}$ and $S_{2}$. The Loewy structures of the projective indecomposable $k A_{4}$-modules are as follows:

$$
P_{0}=S_{1} \begin{gathered}
S_{0} \\
S_{0}
\end{gathered} S_{2}, \quad P_{1}=S_{0} \begin{aligned}
& S_{1} \\
& S_{1}
\end{aligned} S_{2}, \quad P_{2}=S_{1}{ }_{S_{2}} S_{0} .
$$

The principal block $k B$ of $k A_{5}$ also has three simple modules: the trivial simple module $T_{0}$, and two two-dimensional modules $T_{1}$ and $T_{2}$. When considering $T_{1}$ and $T_{2}$ as $k A_{4}$-modules, then $T_{1}$ corresponds to the uniserial module $\begin{aligned} & S_{1} \\ & S_{2}\end{aligned}$, and $T_{2}$ corresponds to the uniserial module $\begin{gathered}S_{2} \\ S_{1}\end{gathered}$. The Loewy structures of the projective indecomposable $k B$-modules are as follows:

$$
\begin{array}{rrrrr} 
& T_{0} & & T_{1} & T_{2} \\
T_{1} & & T_{2} & T_{0} & T_{0} \\
Q_{0}= & T_{0}, & Q_{2}= & T_{2}, \\
T_{2} & & T_{1} & T_{0} & T_{1} \\
T_{2} & T_{1} & T_{2}
\end{array} .
$$

The split-endomorphism two-sided tilting complex $Q_{k}^{\bullet}$ has exactly two non-zero terms, one in degree -1 and one in degree 0 :

$$
Q_{k}^{\bullet}=\cdots \rightarrow 0 \rightarrow X^{-1} \stackrel{d^{-1}}{\longrightarrow} X^{0} \rightarrow 0 \rightarrow \cdots,
$$

where $X^{0}$ is $k B$, considered as a $k B$ - $k A_{4}$-bimodule, $X^{-1}$ is the projective cover, as a bimodule, of the augmentation ideal of $k B$, and the differential $d^{-1}$ is the natural surjection onto the augmentation ideal. It follows from [13, Lemma 3.1] and its proof that, as $k B-k A_{4}$-bimodules,

$$
\begin{aligned}
\operatorname{top}\left(X^{0}\right) & \cong\left(T_{0} \otimes_{k} S_{0}^{*}\right) \oplus\left(T_{1} \otimes_{k} S_{1}^{*}\right) \oplus\left(T_{2} \otimes_{k} S_{2}^{*}\right), \\
X^{-1} & \cong\left(Q_{1} \otimes_{k} P_{1}^{*}\right) \oplus\left(Q_{2} \otimes_{k} P_{2}^{*}\right)
\end{aligned}
$$

where $M^{*}=\operatorname{Hom}_{k}(M, k)$ and $\operatorname{top}\left(X^{0}\right)$ denotes the largest semisimple quotient module of $X^{0}$.

We now want to use Theorem 3.2 to obtain a connection between $R\left(A_{4}, V^{\bullet}\right)$ and $R\left(A_{5}, V^{\mathbf{\bullet}^{\bullet}}\right)$ when $V^{\mathbf{\bullet}^{\bullet}}=Q_{k}^{\bullet} \otimes_{k A_{4}} V^{\bullet}$, in case $V^{\bullet}$ is a one-term complex with a simple $k A_{4}$-module in degree 0 . Since, as left $k B$-modules, $X^{0} \cong Q_{0} \oplus Q_{1}^{2} \oplus Q_{2}^{2}$, it follows that as right $k A_{4}$-modules $X^{0} \cong P_{0}^{*} \oplus P_{1}^{* 5} \oplus P_{2}^{* 5}$. Note that $X^{0}$ induces a stable equivalence between $k B$ and $k A_{4}$. Thus it follows from [9, Thm. 3.4] (see also [16, Lemma 5]) that if $V$ is a simple $k A_{4}$-module, then $X^{0} \otimes_{k A_{4}} V$ is an indecomposable $k B$-module. Moreover, it follows e.g. from the proof of [16, Lemma 2] that, as $k B$ - $k A_{4}$-bimodules,

$$
\operatorname{top}\left(X^{0}\right) \cong \bigoplus_{i=0}^{2} \operatorname{top}\left(X^{0} \otimes_{k A_{4}} S_{i}\right) \otimes_{k} S_{i}^{*},
$$

which implies by (4.1) that $\operatorname{top}\left(X^{0} \otimes_{k A_{4}} S_{i}\right) \cong T_{i}$ for $i \in\{0,1,2\}$. Thus, considering possible indecomposable $k B$-modules with a given top and dimension, it follows 
that, as $k B$-modules,

$$
X^{0} \otimes_{k A_{4}} S_{0} \cong T_{0}, \quad X^{0} \otimes_{k A_{4}} S_{1} \cong \begin{aligned}
& T_{1} \\
& T_{0} \\
& T_{2}
\end{aligned}, \quad X^{0} \otimes_{k A_{4}} S_{2} \cong \begin{aligned}
& T_{2} \\
& T_{0} \\
& T_{1}
\end{aligned} .
$$

Moreover, as $k B$-modules,

$$
X^{-1} \otimes_{k A_{4}} S_{0} \cong 0, \quad X^{-1} \otimes_{k A_{4}} S_{1} \cong Q_{1}, \quad X^{-1} \otimes_{k A_{4}} S_{2} \cong Q_{2} .
$$

Thus, we obtain that if $V_{0}^{\bullet}$ is the one-term complex $S_{0}$, then $V_{0}^{\prime \bullet}$ is the one-term complex $T_{0}$. On the other hand, if $V_{1}^{\bullet}$ is the one-term complex $S_{1}$, then $V_{1}^{\prime \bullet}$ is the complex

$$
V_{1}^{\prime \bullet}=\cdots 0 \rightarrow 0 \rightarrow Q_{1} \stackrel{\pi_{1}}{\longrightarrow} T_{1} T_{0} \rightarrow 0 \rightarrow \cdots,
$$

where $\pi_{1}$ is the natural surjection map. Thus $V_{1}^{\prime \bullet}$ is isomorphic to the one-term complex $\begin{aligned} & T_{0} \\ & T_{1}\end{aligned}$, this time concentrated in degree $(-1)$. Similarly, if $V_{2}^{\bullet}$ is the oneterm complex $S_{2}$, then $V_{2}^{\prime \bullet}$ is isomorphic to the one-term complex $\begin{aligned} & T_{0} \\ & T_{2}\end{aligned}$, concentrated in degree $(-1)$. We conclude from Theorem 3.2 that $R\left(A_{4}, S_{0}\right) \cong R\left(A_{5}, T_{0}\right)$, $R\left(A_{4}, S_{1}\right) \cong R\left(A_{5}, \begin{array}{l}T_{0} \\ T_{1}\end{array}\right)$ and $R\left(A_{4}, S_{2}\right) \cong R\left(A_{5}, \begin{array}{l}T_{0} \\ T_{2}\end{array}\right)$. This coincides with the results found in [1].

\section{REFERENCES}

[1] F. M. Bleher, Universal deformation rings and Klein four defect groups. Trans. Amer. Math. Soc. 354 (2002), 3893-3906. MR1926858 (2004a:20014)

[2] F. M. Bleher and T. Chinburg, Universal deformation rings and cyclic blocks. Math. Ann. 318 (2000), 805-836. MR $1802512(2001 \mathrm{~m}: 20013)$

[3] F. M. Bleher and T. Chinburg, Deformations and derived categories. C. R. Math. Acad. Sci. Paris 334 (2002), 97-100. MR.1885087 (2002k:11077)

[4] F. M. Bleher and T. Chinburg, Deformations and derived categories. To appear in Ann. de l'Institut Fourier (Grenoble).

[5] C. Breuil, B. Conrad, F. Diamond and R. Taylor, On the modularity of elliptic curves over $\mathbb{Q}:$ Wild 3-adic exercises. J. Amer. Math. Soc. 14 (2001), 843-939. MR1839918 (2002d:11058)

[6] M. Broué, Rickard equivalences and block theory. In: Groups 1993, Galway-St. Andrews Conference, vol. 1, London Math. Soc. Lecture Note Ser. 211, Cambridge University Press, Cambridge, 1995, pp. 58-79. MR.1342782 (96d:20011)

[7] G. Cornell, J. H. Silverman and G. Stevens (eds.), Modular Forms and Fermat's Last Theorem (Boston, 1995). Springer-Verlag, Berlin-Heidelberg-New York, 1997. MR1638473 (99k:11004)

[8] B. de Smit and H. W. Lenstra, Explicit construction of universal deformation rings. In: Modular Forms and Fermat's Last Theorem (Boston, MA, 1995), Springer-Verlag, BerlinHeidelberg-New York, 1997, pp. 313-326. MR1638482

[9] M. Linckelmann, The isomorphism problem for cyclic blocks and their source algebras. Invent. Math. 125 (1996), 265-283. MR1395720 (97h:20010)

[10] M. Linckelmann, On splendid derived and stable equivalences between blocks of finite groups. J. Algebra 242 (2001), 819-843. MR.1848975 (2002i:20013)

[11] B. Mazur, Deforming Galois representations. In: Galois groups over $\mathbb{Q}$ (Berkeley, CA, 1987), Springer-Verlag, Berlin-Heidelberg-New York, 1989, pp. 385-437. MR1012172 (90k:11057)

[12] J. Rickard, Derived equivalences as derived functors. J. London Math. Soc. 43 (1991), 37-48. MR:1099084 (92b:16043)

[13] J. Rickard, Splendid equivalences: derived categories and permutation modules. Proc. London Math. Soc. 72 (1996), 331-358. MR:1367082(97b:20011) 
[14] J. Rickard, Triangulated categories in the modular representation theory of finite groups. In: Derived Equivalences for Group Rings, Lecture Notes in Math., 1685, Springer-Verlag, Berlin, 1998, pp. 177-198. MR.1649845

[15] J. Rickard, The abelian defect group conjecture. Proceedings of the International Congress of Mathematicians, Vol. II (Berlin, 1998), Doc. Math., 1998, Extra Vol. II, pp. 121-128. MR.1648062 (99f:20014)

[16] R. Rouquier, From stable equivalences to Rickard equivalences for blocks with cyclic defect. In: Groups 1993, Galway-St. Andrews Conference, vol. 2, London Math. Soc. Lecture Note Ser. 212, Cambridge University Press, Cambridge, 1995, pp. 512-523. MR1337293 (96h:20021)

[17] M. Schlessinger, Functors of Artin Rings. Trans. of the Amer. Math. Soc. 130 (1968), 208-222. MR0217093 (36:184)

[18] R. Taylor and A. Wiles, Ring-theoretic properties of certain Hecke algebras. Ann. of Math. 141 (1995), 553-572. MR1333036 (96d:11072)

[19] A. Wiles, Modular elliptic curves and Fermat's last theorem. Ann. of Math. 141 (1995), 443-551. MR1333035(96d:11071)

Department of Mathematics, University of Iowa, Iowa City, Iowa 52242-1419

E-mail address: fbleher@math.uiowa.edu 